\title{
Hairy Root Technology: Effect of Etiolation on Datura sp. Transgenic Root Induction and Hyoscyamine Production
}

\author{
Boualem HARFI ${ }^{1,2, *}$, Lakhdar KHELIFI ${ }^{2}$
}

\section{Boualem HARFI $1,2, *$, Lakhdar $\mathrm{KHELIFI}^{2}$}

'Biotechnology Research Center (C.R.Bt), Constantine, ALGERIA.

${ }^{2}$ National Higher School of Agronomy (ENSA - El-Harrach), Algiers, ALGERIA.

\section{Correspondence}

\section{Boualem HARFI}

Biotechnology Research Center (C.R.Bt), Constantine, ALGERIA.

Phone no: +231 (0) 317750 44;

Fax: +231 (0) 317750 44;

E-mail: b.harfi@crbt.dz

History

- Submission Date: 30-05-2019;

- Review completed: 09-06-2019;

- Accepted Date: 17-06-2019.

DOI : 10.5530/pj.2019.11.156

Article Available online http://www.phcogj.com/v11/i5

\section{Copyright}

(C) 2019 Phcogi.Com. This is an openaccess article distributed under the terms of the Creative Commons Attribution 4.0 International license.

\begin{abstract}
Introduction: Hyoscyamine, because of its medicinal properties, is an important tropane alkaloid. In order to implement a biotechnological process for its production, hairy roots of Datura species resulting from genetic transformation by Agrobacterium rhizogenes A4 strain have been the subject of this work. In the in vitro alkaloids production programs, optimization of the transformation is a crucial step for obtaining a large number of root lines allowing the selection of efficient lines. Methods: The conditions for hairy roots induction were explored by studying the effect of Datura sp. in vitro seedling etiolation on the genetic transformation. The first step was the establishment of Datura in vitro plantlet cultures followed by the hairy roots induction with $A 4$ strain of $A$. rhizogenes. The confirmation of the genetic transformation was performed by polymerase chain reaction (PCR) analysis of ro/B gene in the roots. After hyoscyamine extraction, it was analyzed (quantitatively and qualitatively) by gas chromatography. Results: 343 root lines were obtained in total, 96 root lines from non-etiolated Datura in vitro seedlings and 247 root lines from etiolated in vitro plantlets. After the selection of six transgenic root lines, tow root lines from each species (D. tramonium, D. tatula and $D$. innoxia), one from non-etiolated in vitro plantlet and one from etiolated in vitro plantlet, the most hyoscyamine content was $8.43 \mathrm{mg} / \mathrm{g}$ D.W. obtained with D. tatula etiolated in vitro seedling. Conclusion The etiolated in vitro plantlets seem more favorable to hairy roots induction.

Key words: Datura sp., etiolation, gas chromatography, hairy roots, hyoscyamine.
\end{abstract}

\section{INTRODUCTION}

Some Solanaceae species including Datura genus biosynthesized in their roots alkaloids. The wild population of Datura species (D. stramonium, $D$. tatula and D. innoxia) that grows in Algeria is particularly rich in hyoscyamine. This last one is a tropane alkaloid used in medicine as useful natural product. ${ }^{1,2}$

In vitro plant cultures drawn an interest and cost-effective alternative to classical approaches to plant secondary metabolite production. They constitute sustainable and eco-friendly system to obtain complex chemical structures biosynthesized by concerned plant species. "Plant Cell Factory" concept defined as a biotechnological systems developed to improve the production of plantderived compounds with biological activity. ${ }^{3}$

Among the plant in vitro production models of bioactive molecules those based on hairy roots techniques. ${ }^{4-6}$ Following plant fresh wound, some soil bacteria are able to transfer some of their genetic information (plasmid) to the nuclear genome of the host plants that are sensitive. This plasmid DNA is then able to express itself. These bacteria are Gram- Rhizobiaceae of Agrobacterium genus. Among of them, A. rhizogenes responsible for hairy root (HR) disease.

HR cultures characterized by a high growth rate and can synthesize secondary metabolites like as original plants roots. Naturally, root cultures require an exogenous supply of plant hormones and have a low growth rate resulting in low biosynthesis of secondary metabolites. However, the use of HR culture has revolutionized plant tissue cultures for secondary metabolites biosynthesis. Their ease of maintenance and their ability to synthesize a wide range of chemical compounds offers an additional advantage as a continuous source of production. ${ }^{8,9}$

Maldonado-Mendosa et al. ${ }^{10}$ have shown a great variability in the rate of growth and alkaloid content between the Datura stramonium root lines. This would result in considerable differences in root growth as well as in the accumulation of secondary metabolites even when they arise from the same transformation event. ${ }^{11,12}$ Hence the interest, in a production program of secondary metabolites based on HR cultures, to induce the maximum number of root lines to increase the probability to select highperforming lines.

The aim of our work was the evaluating the effect of etiolation on hairy roots induction with $A$. rhizogenes in three Datura species. Our hypothesis was that etiolated in vitro seedlings are more sensitive to bacterial diseases than non-etiolated in vitro plantlets (exposed to light), transformation with agrobacteria would be more important (more transgenic roots induced on etiolated explants compared to explants from non-etiolated in vitro seedling). Knowing that root lines are unique, optimization of transformation by $A$. rhizogenes is an essential step for the selection of efficient hairy root. 


\section{MATERIALS AND METHODS}

\section{Establishment of Datura In Vitro plantlet cultures}

Seeds of three Datura species (D. stramonium L., D. tatula L. and D. innoxia Mill.) collected in Mitidja (North of Algeria) constituted the vegetal material of this study. The seeds were scarified and surface disinfected according to Harfi et al. ${ }^{2}$ protocol. They were sown in test tubes containing $20 \mathrm{ml}$ of solid hormone-free Murashige and Skoog (MS) medium (Sigma, USA) containing $2 \%$ sucrose, $0.7 \%$ agar and $\mathrm{pH}$ adjusted at $5.6-5.8$.

For each species, tow plots of 16 test tubes were constituted. The first plot represents the control group (non-etiolated in vitro plantlets) and were cultivated at $26{ }^{\circ} \mathrm{C} \pm 1{ }^{\circ} \mathrm{C}$ with a daily 16 h-light photoperiod. The second one represents the group of etiolated in vitro plantlets and were cultivated at $26^{\circ} \mathrm{C} \pm 1{ }^{\circ} \mathrm{C}$ with total darkness. The experiment was repeated three times. For each species and for each culture conditions we used 48 test tubes. In total, 288 test tubes were used.

\section{Establishment of hairy root cultures}

Hairy roots (HRs) cultures were obtained from hypocotyl fragments of 0.5 to $1 \mathrm{~cm}$ taken on the Datura sp. in vitro seedlings 45 days old via genetic transformation with Agrobacterium rhizogenes as described by Harfi et al. ${ }^{5}$. The used A4 strain of $A$. rhizogenes is an agropine-type plasmid. The co-culture (bacteria - explant) was done by simple deposit using a syringe on the level of the basal section of the hypocotyls. The culture of hypocotyls is operated on MS solid medium containing 250 $\mathrm{mg} / \mathrm{L}$ cefotaxime. Only emergent roots from the infection site were retained. Root segments were excised when they reach approximately $2 \mathrm{~cm}$ length. HRs multiplication were carried out on fresh B5 solid medium without antibiotic and were cut off in $2 \mathrm{~cm}$ long segments.

The effect of seedling etiolation on HRs induction was measured according the three main following criteria:

- Time of appearance of the first root (day);

- Average number of roots per explant;

- Total obtained root (from 48 explants) per species.

\section{Confirmation of transformation and selection of efficient root lines}

Genetic transformation with A4 strain of A. rhizogenes was confirmed by the presence of $r o l$ genes $(r o l B)$ in HRs using PCR (Polymerase Chain Reaction). ${ }^{2}$

The selection of the efficient root lines was based on two main criteria:

- HR biomass ( $\mathrm{mg} / 50 \mathrm{ml}$ of B5 liquid medium after 20 days of culture);

- Hyoscyamine content (mg / g D.W.).

\section{Tropane alkaloids extraction}

Hyoscyamine extraction was carried out according to Amdoun et al. ${ }^{13}$ protocol. HRs samples of $50 \mathrm{mg}$ dried for $48 \mathrm{~h}$ at $40^{\circ} \mathrm{C}$ and powdered, tropane alkaloids were extracted with $\mathrm{HCl} 0.1 \mathrm{~N}$ solution. $\mathrm{NH}_{4} \mathrm{OH}$ was used to reach $\mathrm{pH}$ 10. Aqueous phase was filtered and extracted three times with an equal volume of chloroform. The organic phase was evaporated and the residue was suspended in dichloromethane.

\section{Gas chromatography analysis}

Gas chromatography (GC) was performed on a CHROMPACK CP 9002 (Chrompack International B.V., Middelburg, The Netherlands), equipped with a flame ionization detector (FID). A fractionated sample of $2 \mu \mathrm{L}$ was injected into a capillary DB1 column. The used GC conditions were as described by Harfi et al. ${ }^{5}$ (carrier gas: nitrogen; Combustion gas: hydrogen; detector temperature: $260{ }^{\circ} \mathrm{C}$; injection temperature: $260^{\circ} \mathrm{C}$ and oven temperature: $250{ }^{\circ} \mathrm{C}$ ). Hyoscyamine identification and quantification were performed using a standard prepared under the same conditions as the samples. Each sample analysis was repeated in triplicate.

\section{Statistical analysis}

After data homogeneity and normality tests, statistical analysis was performed. It was carried out with SPSS software version 20.0 according to variance analysis (one-way ANOVA) and Tukey's test for the obtained results. The values followed by the same letter are not significantly different from each other (P 0.05: level of significance mean).

\section{RESULTS}

The interest of our work was to evaluate the effect of in vitro seedlings etiolation on hairy roots induction of three Datura species using $A$. rhizogenes. The aim was to improve the number of induced root lines in order to increase the probability of selecting efficient lines in terms of hyoscyamine and biomass productions. Figure 1 illustrates the experimental protocol steps adopted from the establishment of in vitro seedlings (etiolated and non-etiolated) until the selection of efficient root lines.

The statistical study reveals a significant effect of seedling etiolation on hairy roots induction (Table 1). For the mean time of appearance of the first root, the most interesting homogeneous group (a) is represented by etiolated in vitro seedlings of $D$. tatula explants with a mean time of 7.92 days. It was with the explants of $D$. starmonium etiolated seedlings that we recorded the statistically highest average number of transgenic roots per explant (2.62). Also, the total number of root lines obtained from 48 explants was greater in etiolated seedlings than in non-etiolated in vitro seedlings. There were 126,93 and 28 root lines respectively for the explants of the etiolated in vitro seedlings of $D$. stramonium, D. tatula and $D$. innoxia and 54, 31 and 11 root lines respectively for the explants of non-etiolated in vitro seedlings of $D$. stramonium, D. tatula and $D$. innoxia. Explants from etiolated in vitro plantlets show the best genetic transformation capacities compared to non-etiolated explants.

On the whole of all obtained root lines from the three Datura species (343 lines in total, 96 root lines from non-etiolated in vitro seedlings and 247 root lines from etiolated in vitro plantlets), six root lines were retained on the basis of their hyoscyamine content (Table 2). For each species, two root lines were retained, one from the etiolated in vitro seedlings and one from the non-etiolated in vitro plantlets.

Table 1: Effect of in vitro seedlings etiolation on Datura sp. hairy roots induction.

\begin{tabular}{lcccccc}
\hline & \multicolumn{3}{c}{ Non-etiolated in vitro seedling } & \multicolumn{3}{c}{ Etiolated in vitro seedling } \\
\cline { 2 - 7 } & DS & DT & DI & DS & DT & DI \\
\hline Time of appearance of the first root (d) & $16.58^{\mathrm{c}}$ & $15.24^{\mathrm{c}}$ & $16,97^{\mathrm{c}}$ & $8.75^{\mathrm{a}}$ & $7.92^{\mathrm{a}}$ & $11.02^{\mathrm{b}}$ \\
Average number of roots per explant & $1.12^{\mathrm{C}}$ & $0.64^{\mathrm{D}}$ & $0,23^{\mathrm{E}}$ & $2.62^{\mathrm{A}}$ & $1.94^{\mathrm{B}}$ & $0.58^{\mathrm{D}}$ \\
Total obtained root (from 48 explants) & 54 & 31 & 11 & 126 & 93 & 28 \\
\hline
\end{tabular}

The values are given as the mean $\pm \mathrm{SD}$, different letters indicate significant difference between values according to ANOVA test (p: 0.05); d: days; DS: Datura stramonium in vitro seedlings; DT: D. tatula in vitro seedlings; DI: D. innoxia in vitro seedlings. 


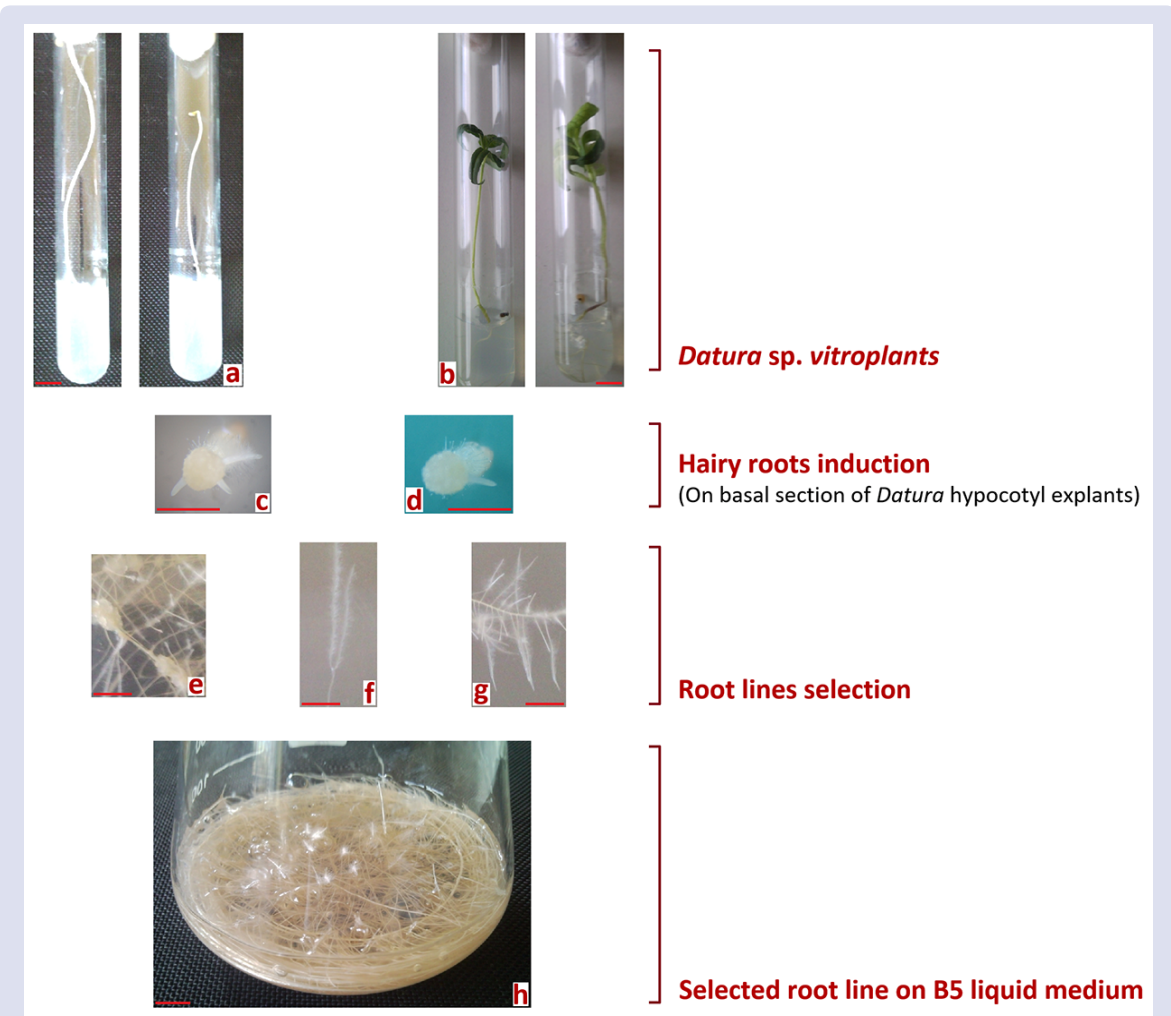

Figure 1: Hairy root culture establishment from Datura sp. etiolated and non-etiolated in vitro seedling.

a: Datura sp. etiolated in vitro seedlings; b: Datura sp. non-etiolated in vitro seedlings; c: transgenic roots induction (3 roots) on hypocotyl from Datura sp. etiolated in vitro seedlings; $\mathbf{d}$ : transgenic root induction (1 root) on hypocotyl from Datura sp. non-etiolated in vitro seedlings; e: root line with callus formation; $\mathbf{f}$ : root line with low growth rate; $\mathbf{g}$ : root line with high growth rate and good ramification level (selected root line); $\mathbf{h}$ : hairy roots culture on B5 liquid medium.

Table 2: Hyoscyamine content of selected root lines from non-etiolated / etiolated Datura sp. in vitro seedlings.

\begin{tabular}{ccc}
\hline Datura sp. in vitro seedling & Selected root lines & Hyoscyamine content (mg/g D.W.) \\
\hline \multirow{3}{*}{ Non-etiolated } & NE- $\mathrm{L}_{\text {DS }}$ & $8.18 \pm 0.54^{\mathrm{a}}$ \\
& NE- $\mathrm{L}_{\mathrm{DT}}$ & $6.06 \pm 0.38^{\mathrm{b}}$ \\
& NE- $\mathrm{L}_{\mathrm{DI}}$ & $2.01 \pm 0.20^{\mathrm{d}}$ \\
\multirow{2}{*}{ Etiolated } & E- $\mathrm{L}_{\mathrm{DS}}$ & $8.21 \pm 0.72^{\mathrm{a}}$ \\
& $\mathrm{E}-\mathrm{L}_{\mathrm{DT}}$ & $8.43 \pm 0.42^{\mathrm{a}}$ \\
& E- $\mathrm{L}_{\mathrm{DI}}$ & $3.04 \pm 0.27^{\mathrm{c}}$ \\
\hline
\end{tabular}

The values are given as the mean $\pm \mathrm{SD}$, different letters indicate significant difference between values according to ANOVA test (p: 0.05); D.W.: Dry weight; NE- $\mathbf{L}_{\mathrm{Ds}}$ : Selected root line from non-etiolated Datura stramonium in vitro seedlings; $\mathbf{N E}-\mathrm{L}_{\mathrm{DT}}$ : Selected root line from non-etiolated $D$. tatula in vitro seedlings; NE- $\mathbf{L}_{\mathrm{DI}}$ : Selected root line from non-etiolated D. innoxia in vitro seedlings; $\mathbf{E}-\mathrm{L}_{\mathrm{Ds}}$ : Selected root line from etiolated $D$. stramonium in vitro seedlings; $\mathbf{E}-\mathbf{L}_{\mathrm{DT}}$ : Selected root line from etiolated $D$. tatula in vitro seedlings; $\mathbf{E}-\mathbf{L}_{\mathrm{Dr}}$ : Selected root line from etiolated $D$. innoxia in vitro seedlings.

The statistical study of etiolation on hyoscyamine content of the six retained root lines reveals a significant effect of the etiolation on the tropane alkaloid content for D. tatula and D. innoxia species. However, for the D. stramonium, etiolation of in vitro plantlets did not affect the hyoscyamine content. This may be explained by the number of induced root lines with non-etiolated $D$. starmonium explants that is relatively high (54 lines), which has made it possible to select a root line with a high hyoscyamine content.

\section{DISCUSSION}

The development of genetically transformed plant tissue cultures and mainly HRs induced by $A$. rhizogenes is a key step in the use of in vitro cultures for the production of secondary metabolites. HRs can be a promising source for standardized production of secondary metabolites. It is generally admitted that HRs produce secondary metabolites generation after generation without loss of genetic and biosynthetic stabilities. ${ }^{14,15}$

The etiolated in vitro seedlings are characterized by an absence of chlorophyll in the tissues and an excessive elongation of the plantlets. Light is a key factor for the growth, development and response to plant stress. The plant cell is equipped with "light-signal" mechanisms located within the chloroplast that is involved in the defense of the plant. Light perception and stress response could work and act together to form a plant defense network. ${ }^{16}$ According to Yaeno et al. ${ }^{17}$ trienoic fatty acids in chloroplast lipid membrane are decisive for adaptation to stress and play an important role in the regulation of the plant's defense against external aggressions (biotic and abiotic stresses). In addition, 
the reinforcement of structural barriers with lignin and suberin at the cellular level is also a common response in plants exposed to ultraviolet light. ${ }^{18}$

Indeed, the improvement of the results of the HRs induction by $A$. rhizogenes with the explants from etiolated in vitro seedlings can be explained by the fragility of etiolated plantlets, they were more susceptible to microbial attack than non-etiolated ones. This sensitivity would induce a more favorable response to the transformation by the A. rhizogenes.

In the spontaneous whole plants of Datura species the mean hyoscyamine contents are between $0.2-0.8 \mathrm{mg} / \mathrm{g}$ D.W. ${ }^{1}$. Also, the average hyoscyamine levels of the HRs of these species are $2.1 \mathrm{mg} / \mathrm{g}$ D.W. and $1.7 \mathrm{mg} / \mathrm{g}$ D.W. respectively for $D$. stramonium ${ }^{12}$ and $D$. innoxia $^{19}$. The best hyoscyamine contents obtained in our study were $8.21 \mathrm{mg} / \mathrm{g}$ D.W. for selected root line from D. stramonium, $8.43 \mathrm{mg} / \mathrm{g}$ D.W. for selected root line from D. tatula and $3.04 \mathrm{mg} / \mathrm{g}$ D.W. for selected root line from $D$. innoxia. Our results compared to those cited in the bibliography show the interest of inducing a maximum of transgenic root lines in order to select lines with an important hyoscyamine contents. According to Zhi-Bi and $\mathrm{Min}^{11}$ and Georgiev et al. ${ }^{12}$, each root line obtained following transformation by A. rhizogenes can be considered a different line from all others on a genetic and biochemical level. Thus, in the production programs of alkaloids via in vitro plant culture technics, the optimization of the transformation by the $A$. rhizogenes is a crucial and unavoidable step for obtaining a large number of HRs allowing the selection of efficient lines.

\section{CONCLUSION}

HRs cultures are characterized by a high growth rate and can synthesize secondary metabolites in the same way as the roots of the original plants. Naturally, root cultures require an exogenous supply of plant hormones and have a very slow growth rate resulting in low secondary metabolites synthesis. However, the use of HR cultures has revolutionized the plant tissues cultures for the biosynthesis of secondary metabolites. Their ease maintenance and their ability to synthesize a wide range of chemical compounds offers an additional advantage as a continuous source of production of secondary metabolites. ${ }^{8,9}$

From all obtained results, we confirmed our initial hypothesis "etiolated in vitro seedlings are more sensitive to hairy root disease than non-etiolated in vitro plantlets". Our study confirms the interest of improving root lines induction using genetic transformation with $A$. rhizogens in program drawn up on hairy root technology for a plant secondary metabolites production.

\section{ACKNOWLEDGEMENTS}

This work has been carried out at the Laboratory of Genetic Resources and Biotechnology (L-RGB) of the National Higher School of Agronomy (ENSA - El-Harrach), Algiers, ALGERIA.

\section{CONFLICTS OF INTEREST}

The authors declare no conflicts of interest.

\section{REFERENCES}

1. Houmani Z, Cosson L, Corbineau F, Côme D. Etude de la teneur en hyoscyamine et scopolamine d'une population sauvage de Datura stramonium L. en Algérie. Acta Bot Gallica. 1994;141:61-6.

2. Harfi B, Khelifi-Slaoui M, Bekhouche M, Benyammi R, Hefferon K, Makhzoum A et al. Hyoscyamine production in hairy roots of three Datura species exposed to high-salt medium. In Vitro Cell Dev Biol - Plant. 2016;52:92-8.

3. Ramirez-Estrada K, Vidal-Limon H, Hidalgo D, Moyano E, Golenioswki M, Cusidó RM, et al. Elicitation, an effective strategy for the biotechnological production of bioactive high-added value compounds in plant cell factories. Molecules. 2016;21:182.

4. Benyammi R, Paris C, Khelifi-Slaoui M, Zaoui D, Belabbassi O, Bakiri N, et al. Screening and kinetic studies of catharanthine and ajmalicine accumulation and their correlation with growth biomass in Catharanthus roseus hairy roots. Pharm Biol. 2016;54:2033-43.

5. Harfi B, Khelifi L, Khelifi-Slaoui M, Assaf-Ducrocq C, Gontier E. Tropane alkaloids GC/MS analysis and low dose elicitors' effects on hyoscyamine biosynthetic pathway in hairy roots of Algerian Datura species. Sci Rep. 2018;8:17951.

6. Amdoun R, Benyoussef E-H, Benamghar A, Khelifi L. Prediction of hyoscyamine content in Datura stramonium L. hairy roots using different modeling approaches: Response Surface Methodology (RSM), Artificial Neural Network (ANN) and Kriging. Biochem Eng J. 2019;144:8-17.

7. Mehrotra S, Srivastava V, Rahman LU, Kukreja AK. Hairy root biotechnologyindicative timeline to understand missing links and future outlook. Protoplasma. 2015;252:1189-201.

8. Hamill JD, Parr AJ, Robins RJ, Rhodes MJC. Secondary product formation by cultures of Beta vulgaris and Nicotiana rustica transformed with Agrobacterium rhizogenes. Plant Cell Rep. 1986;5:111-4.

9. Verma PC, ur Rahman L, Negi AS, Jain DC, Khanuja SPS, Banerjee S Agrobacterium rhizogenes-mediated transformation of Picrorhiza kurroa Royle ex Benth.: establishment and selection of superior hairy root clone. Plant Biotechnol Rep. 2007;1:169-174.

10. Maldonado-Mendoza IE, Ayora-Talavera T, Loyola-Vargas VM. Establishment of hairy root cultures of Datura stramonium: Characterization and stability of tropane alkaloid production during long periods of subculturing. Plant Cell Tiss Organ Cult. 1993;33:321-9

11. Zhi-Bi H, Min D. Hairy roots and its application in plant genetic engineering. $J$ Integr Plant Biol. 2006;48:121-7.

12. Georgiev MI, Pavlov Al, BleyT. Hairy root type plant in vitro systems as sources of bioactive substances. Appl Microbiol Biotechnol. 2007:74:1175-85.

13. Amdoun R, Khelifi L, Khelifi-Slaoui M, Amroune S, Asch M, Assaf-Ducrocq C, et al. Optimization of the culture medium composition to improve the production of hyoscyamine in elicited Datura stramonium $\mathrm{L}$. hairy roots using the response surface methodology (RSM). Int J Mol Sci. 2010;11:4726-40.

14. Giri A, Narasu ML. Transgenic hairy roots: recent trends and applications. Biotechnol Adv. 2000;18:1-22

15. Veena V, Taylor CG. Agrobacterium rhizogenes: recent developments and promising applications. In Vitro Cell Dev Biol - Plant. 2007;43:383-403.

16. Karpinski S, Gabrys H, Mateo A, Karpinska B, Mullineaux PM. Light perception in plant disease defense signaling. Curr Opin Plant Biol. 2003;6:390-6.

17. Yaeno $T$, Matsuda $\mathrm{O}$, Iba K. Role of chloroplast trienoic fatty acids in plant disease defense responses. Plant J. 2004;40:931-41.

18. Charles MT, Goulet A, Arul J. Physiological basis of UV-C induced resistance to Botrytis cinerea in tomato fruit: IV. Biochemical modification of structural barriers. Postharvest Biol Technol. 2008;47:41-53.

19. Shimomura K, Sauerwein M, Ishimaru K. Tropane alkaloids in adventitious and hairy root cultures of Solanaceous plants. Phytochemistry. 1991;30:2275-8. 


\section{GRAPHICAL ABSTRACT}

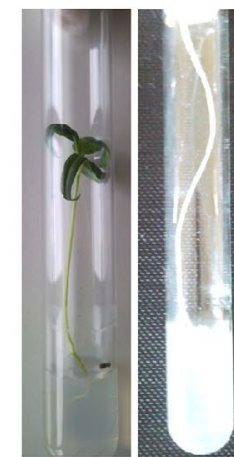

\section{Hairy roots induction}

With Agrobacterium rhizogenes

Datura sp. in vitro seedling (non-etiolated and etiolated)

\section{ABOUT AUTHORS}

Dr. Boualem HARFI, Director of the research division "Biotechnology and Agriculture" at the Biotechnology Research Center (C.R.Bt - Constantine, ALGERIA) since 2014. He completed his studies in agronomic sciences at the National Higher School of Agronomy (ENSA - El-Harrach) and earned his doctorate in 2013 with emphasis in "Plant Biotechnologies". His work focuses on the development of biotechnological models based on plant tissue culture to produce biologically active molecules from plant.

Professor Lakhdar KHELIFI, Director of the National Higher School of Agronomy (ENSA - El-Harrach, ALGERIA). He earned his Ph. D. degree in "Plant Biotechnologies" in 1990. From 2001 to 2015, he was the head of the national research laboratory "Genetic Resources and Biotechnology - LRGB". His work is mainly focused on the protection, valorization and improvement of local genetic resources.

Cite this article: HARFI B, KHELIFI L. Hairy Root Technology: Effect of Etiolation on Datura sp. Transgenic Root Induction and Hyoscyamine Production. Pharmacog J. 2019;11(5):991-5. 